\title{
Contagem de plaquetas: avaliação de metodologias manuais e aplicação na rotina laboratorial
}

\section{Platelet count: evaluation of manual methodologies and application in the laboratory routine}

Samuel R. Comar ${ }^{l}$

Heloísa S. M. Danchura ${ }^{2}$

Paulo H. Silva ${ }^{3}$
A contagem de plaquetas por microscopia é uma ferramenta fundamental na avaliação dos pacientes com doenças que acometem as plaquetas, na medida em que complementa e corrobora as informações fornecidas pelos analisadores hematológicos, além de servir de ferramenta de controle interno da qualidade das contagens automatizadas de plaquetas. Oobjetivo deste trabalho foi comparar quatro métodos manuais de estimativa microscópica da contagem de plaquetas, considerando a correlação com a contagem automatizada de plaquetas e a correlação entre um método e outro, além de fornecer subsídios para a validação dos mesmos nos laboratórios de hematologia. Foram avaliados, neste trabalho, o método de Fonio modificado, o de Bárbara H. O'Connor, o de Nosanchuk, Chang \& Bennett e um método alternativo baseado em um cálculo envolvendo a hemoglobina do paciente. Todos obtiveram boa correlação estatística quando comparados com a contagem automatizada de plaquetas e entre um e outro, sugerindo que o seu uso pode ser recomendado na avaliação plaquetária de pacientes com os mais diversos valores de contagem de plaquetas. Rev. Bras. Hematol. Hemoter.

Palavras-chave: Avaliação plaquetária em lâmina; contagem de plaquetas; controle de qualidade; trombocitopenia; trombocitose.

\section{Introdução}

A plaqueta sanguínea, que possui formato discoide e estrutura altamente complexa, é um fragmento do citoplasma do megacariócito com enorme conteúdo energético e que desempenha funções hemostáticas insubstituíveis. ${ }^{1} \mathrm{~A}$ descrição das plaquetas como corpúsculos distintos do sangue e seu envolvimento no mecanismo de coagulação sanguínea in vivo foi primeiramente descrito pelo patologista italiano Giulio Bizzozero em 1882. ${ }^{2}$ As plaquetas circulam na corrente sanguínea durante sete a dez dias e quase um trilhão delas circula em um humano adulto, o qual produz aproximadamente 100 bilhões de plaquetas por dia. ${ }^{3}$
Trombocitopenia define circunstâncias associadas com a circulação diminuída das plaquetas e são mais comuns do que aquelas associadas com circulação aumentada das mesmas, situação conhecida como trombocitose. Considerando que estados trombocitopênicos são associados frequentemente com sangramento mucocutâneo, a trombocitose pode ser associada com risco trombótico aumentado, particularmente na circulação arterial. ${ }^{1}$

A contagem de plaquetas por microscopia (CPM) é uma ferramenta fundamental na avaliação dos pacientes com doenças que acometem as plaquetas na medida em que adiciona informações úteis àquelas obtidas pelos analisadores hematológicos, além de servir de ferramenta de

\footnotetext{
${ }_{2}^{1}$ Farmacêutico-bioquímico. Seção de Hematologia da Unidade de Apoio Diagnóstico do Hospital de Clínicas da UFPR - Curitiba, PR Farmacêutica-bioquímica e industrial. Schering-Plough Produtos Farmacêuticos Ltda.

${ }^{3}$ Farmacêutico-bioquímico. Professor Adjunto I da disciplina de Hematologia II do curso de Farmácia da UFPR - Curitiba, PR.

Universidade Federal do Paraná (UFPR) - Curitiba, PR.

Correspondência: Samuel Ricardo Comar

Seção de Hematologia da Unidade de Apoio Diagnóstico do Hospital de Clínicas da Universidade Federal do Paraná - HC-UFPR.

Rua Padre Camargo, 280 - Alto da Glória

80060-240 - Curitiba-PR - Brasil

Tel.: (+55 41) 3360-7973

E-mail:srcomar@ufpr.br
} 
controle interno da qualidade das contagens automatizadas de plaquetas (CAP). ${ }^{4,5}$

Para se realizar as CPM, as lâminas de sangue periférico são preparadas a partir de sangue capilar ou venoso, com ou sem a adição de anticoagulante. Em uma lâmina devidamente bem corada, as plaquetas possuem cor púrpura devido ao conteúdo dos grânulos alfa que incluem uma variedade de proteínas de adesão e fatores e inibidores da coagulação. Em alguns laboratórios de hematologia, que utilizam analisadores hematológicos modernos, nos quais a implantação manual em seu software de limites de distinção entre amostras possivelmente normais ou com alterações hematológicas significativas permite fazer uma triagem para definir em quais amostras as extensões sanguíneas necessitarão de análise microscópica, somente amostras de sangue colhidas em ácido etilenodiamino tetra-acético (EDTA) são usadas para se confeccionarem as lâminas. E quando se utilizam amostras de sangue colhidas com anticoagulante, as extensões sanguíneas devem ser preparadas e coradas dentro de duas e quatro horas após a coleta para evitar a ocorrência de artefatos na coloração e na morfologia plaquetária. ${ }^{4-8}$

Apesar da CAP ser mais precisa e exata que a CPM, há um risco potencial para contagens falsamente baixas ou altas nos analisadores hematológicos. Assim, agregados plaquetários, macroplaquetas e plaquetas gigantes podem conduzir a resultados falsamente diminuídos na CAP ao passo que fragmentos eritrocitários, fragmentos citoplasmáticos de células leucêmicas, micrócitos com volume próximo ao limite de corte, lipemia, bactérias e leveduras podem conduzir a resultados falsamente aumentados, o que torna a CPM uma ferramenta essencial para identificar estas causas de contagens espúrias de plaquetas. ${ }^{4,9,10} \mathrm{O}$ objetivo deste trabalho foi comparar métodos manuais de CPM considerando a correlação com a CAP e a correlação entre um método e outro, além de fornecer subsídios para a validação destes métodos de contagem de plaquetas em laboratórios de hematologia.

\section{Casuística e Método}

Amostra - Amostras de sangue total de 101 pacientes, para os quais o hemograma foi solicitado, foram coletadas em tubos Vacutainer contendo $\mathrm{K}_{3}$ EDTA. As extensões sanguíneas foram confeccionadas a partir da amostra com anticoagulante com o auxílio de uma lâmina extensora de modo que não houvesse tangenciamento da porção lateral das extensões sanguíneas com as bordas da lâmina. Em seguida, as lâminas foram coradas pelo método de May Grünwald-Giemsa. A dosagem de hemoglobina e as contagens automatizadas de eritrócitos e plaquetas foram realizadas no analisador hematológico Cell-Dyn 3000 (Abbott Diagnostics, EUA).

Considerações a respeito das CPM realizadas neste trabalho - As CPM foram realizadas utilizando-se um microscópio ótico Eclipse E200 (Nikon Corporation, Japão) com lente de imersão com aumento de 100x em conjunto com uma ocular de campo amplo com aumento de 10x, totalizando um aumento de 1000x. Na porção da lâmina onde normalmente se faz a avaliação qualitativa dos eritrócitos, na qual se observa confinamento dos mesmos, porém sem sobreposição celular, tanto as bordas como a porção central desta região foram examinadas. Foram enumeradas plaquetas em cada uma das 101 extensões sanguíneas. As lâminas foram examinadas sem o conhecimento prévio dos resultados fornecidos pelo analisador hematológico.

Estimativa plaquetária pelo método de Fonio modificado-Diferentemente do método original que emprega sulfato de magnésio a 14\% para evitar agregação plaquetária do sangue obtido por punção digital, ${ }^{11}$ foram utilizadas neste trabalho extensões sanguíneas confeccionadas a partir de amostras de sangue total coletadas em $\mathrm{K}_{3}$ EDTA. Deste ponto em diante foi seguida a técnica original, na qual, para se realizar a CPM, foram enumeradas no microscópio ótico, com aumento de 1000x, plaquetas em 1000 eritrócitos e, em seguida, foi calculado o número de plaquetas da seguinte forma: Plaquetas $/ \mu 1=$ Número de plaquetas enumeradas $\mathrm{x}$ Número de eritrócitos/ $\mu 1 / 1000$.

Estimativa plaquetária pelo método de Bárbara $H$. $O^{\prime}$ Connor - Para se fazer a CPM por este método, foram enumeradas no microscópio ótico com aumento de $1000 \mathrm{x}$, plaquetas em 10 campos e, em seguida, calculou-se o valor médio por campo. Este valor foi multiplicado por 13000 (constante de multiplicação) para fornecer o valor estimado da contagem de plaquetas. ${ }^{8}$

Estimativa plaquetária pelo método de Nosanchuk, Chang \& Bennett - Para se fazer a CPM por este método, foram enumeradas no microscópio ótico com aumento de $1000 x$, plaquetas em 8 campos. A soma obtida foi multiplicada por 2000 (constante de multiplicação) para fornecer o valor estimado de plaquetas por microlitro de sangue. ${ }^{5}$

Método alternativo de estimativa plaquetária em lâmina - Foram enumeradas no microscópio ótico com aumento de $1000 \mathrm{x}$, plaquetas em 10 campos e, em seguida calculouse o valor médio por campo. Multiplicou-se o valor obtido pela hemoglobina do paciente em $\mathrm{g} / \mathrm{dl}$ e em seguida multiplicou-se por 1000 para fornecer o valor da CPM por microlitro de sangue. ${ }^{12}$

Análises estatísticas - Os resultados obtidos foram analisados estatisticamente empregando-se o programa Excel (Microsoft Corporation, EUA). A significância estatística dos dados experimentais foi analisada pelo teste $t$ presumindo variâncias diferentes, onde valores de $\mathrm{p} \leq 0,05$ foram considerados como significativos. A correlação entre os métodos de CPM e a CAP e entre dois métodos de CPM foi realizada 

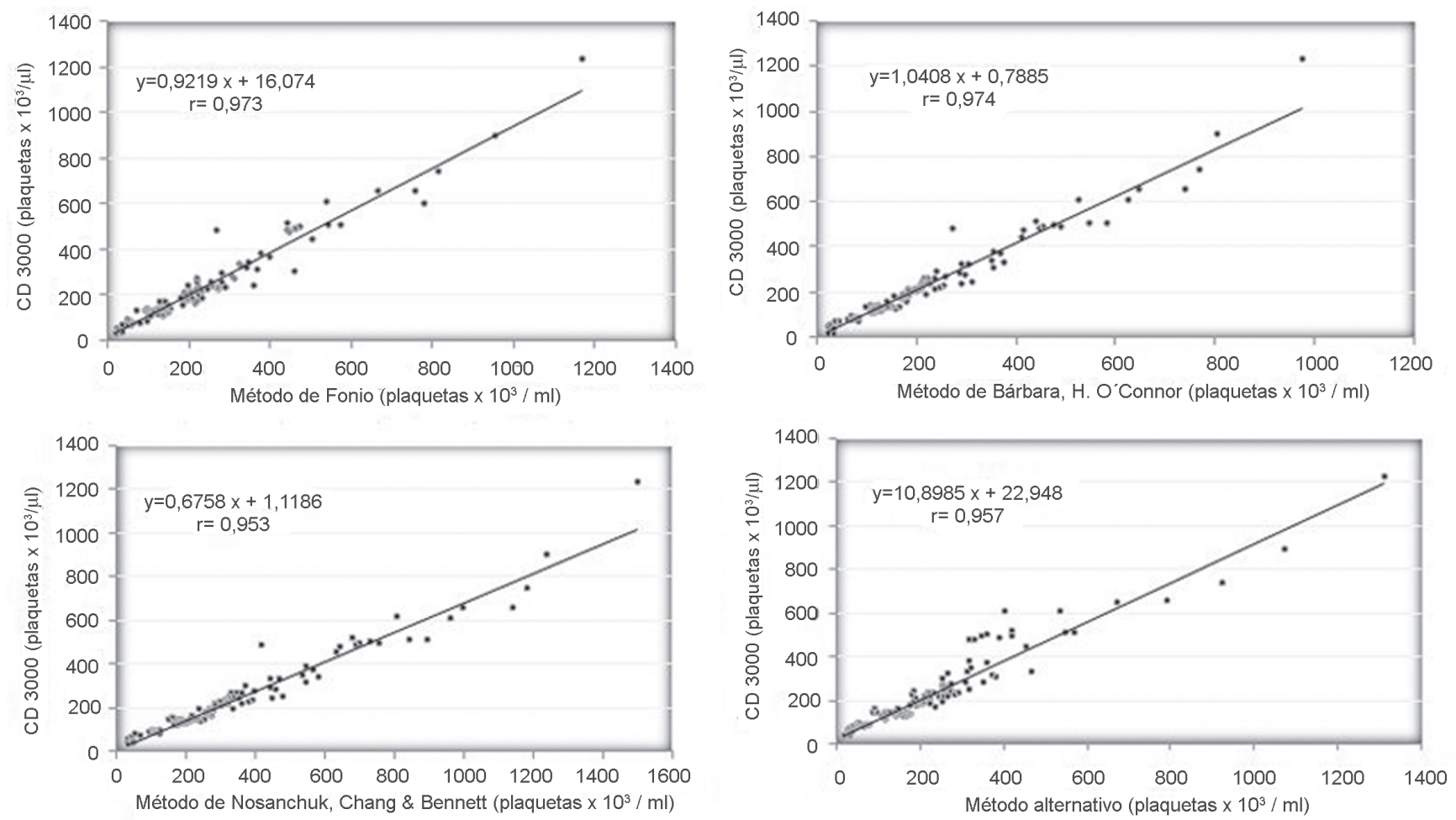

Figura 1. Correlação entre a contagem automatizada de plaquetas e os métodos manuais de estimativa microscópica da contagem de plaquetas. $n=101$ amostras

por análise de regressão, onde $r \geq 0,90$ foi considerado como bom índice de correlação. A correlação foi verificada utilizando-se contagens plaquetárias altas, baixas e normais.

\section{Resultados}

A Figura 1 mostra a relação entre o numero de plaquetas das 101 amostras contadas pelo analisador hematológico Cell Dyn 3000 e pelos métodos manuais de Fonio, Bárbara H. O'Connor, Nosanchuk, Chang \& Bennett e método alternativo baseado no valor da hemoglobina do paciente. As amostras estudadas obtiveram contagens que indicaram desde trombocitopenia a trombocitose. A análise de regressão realizada entre as metodologias forneceu o coeficiente de correlação $(r)$ e a equação de correlação $(y=a x+b)$, onde y corresponde ao número de plaquetas pelo método automatizado e $\mathrm{x}$ ao número de plaquetas pelo método manual. A letra a representa a inclinação da reta e a letra $\mathrm{b}$ o ponto em que a reta cortou o eixo das ordenadas.

A Figura 2 mostra a relação entre o número de plaquetas quantificadas em 10 campos microscópicos com aumento de 1000x utilizando oculares de campo amplo e o número de plaquetas contadas pelo analisador hematológico Cell Dyn 3000 em amostras de sangue de 101 pacientes. A análise de regressão linear deste grupo mostrou uma inclinação de 1317,5 , ponto de interceptação do eixo y de 11176 e um coeficiente de correlação linear de 0,950 . Assim, a fórmula pode

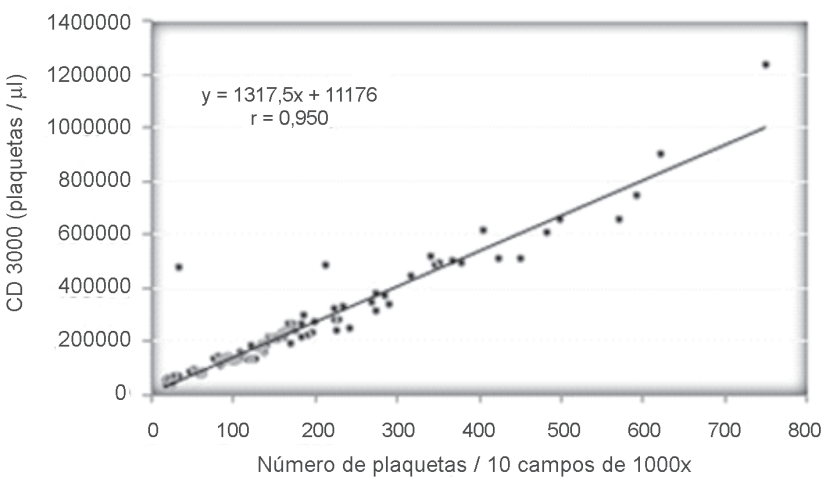

Figura 2. Correlação entre o número de plaquetas enumeradas em 10 campos microscópicos com magnificação de $1.000 x$ e a contagem automatizada de plaquetas. $n=101$ amostras

ser expressa como $\mathrm{y}=1317,5 \mathrm{x}+11176$, onde $\mathrm{x}$ é o número de plaquetas em 10 campos microscópicos e y é o número de plaquetas contadas eletronicamente. Para derivar uma fórmula de trabalho prática, o fator de $\mathrm{y}=1.380 \mathrm{x}$ foi selecionado. Por exemplo, se uma extensão sanguínea de determinado paciente apresentar 250 plaquetas em dez campos microscópicos, o valor estimado da contagem de plaquetas é 1.380 × 250 ou 345.000/ $\mu 1$. Pode-se também calcular a média dos dez campos e multiplicar por 13.800. A Tabela 1 mostra a análise estatística da comparação entre os métodos manuais de CPM com as CAP. Não se observaram diferenças estatisticamente 


\begin{tabular}{|c|c|c|}
\hline \multicolumn{3}{|c|}{$\begin{array}{l}\text { Tabela 1. Dados estatísticos obtidos na avaliação das } \\
\text { metodologias de contagem de plaquetas }\end{array}$} \\
\hline Métodos comparados & Valor de $p$ & Valor de $r$ \\
\hline Fonio e Cell Dyn 3000 & 0,890 & 0,973 \\
\hline Bárbara H. O'Connor e Cell Dyn 3000 & 0,688 & 0,974 \\
\hline $\begin{array}{l}\text { Nosanchuk, Chang \& Bennett } \\
\text { Cell Dyn } 3000\end{array}$ & 0,149 & 0,953 \\
\hline Método alternativo e Cell Dyn 3000 & 0,919 & 0,957 \\
\hline Fonio e Bárbara H. O' Connor & 0,596 & 0,987 \\
\hline Fonio e Nosanchuk, Chang \& Bennet & 0,202 & 0,973 \\
\hline Fonio e método alternativo & 0,972 & 0,964 \\
\hline $\begin{array}{l}\text { Bárbara H. O Connor e Nosanchuk, } \\
\text { Chang \& Bennet }\end{array}$ & 0,063 & 0,992 \\
\hline Bárbara H. O' Connor e método alternativo & 0,623 & 0,963 \\
\hline $\begin{array}{l}\text { Nosanchuk, Chang \& Bennet e método } \\
\text { alternativo }\end{array}$ & 0,192 & 0,940 \\
\hline
\end{tabular}

Análises realizadas: Teste t e análise de regressão

significativas $(\mathrm{p} \leq 0,05)$ entre os métodos analisados pelo Teste t e todas as correlações mostraram valores de $r>0,90$.

\section{Discussão}

A CPM é uma ferramenta coadjuvante extremamente útil no exame hematológico dos pacientes, sobretudo nos serviços de hematologia e oncologia onde contagens de plaquetas são frequentemente solicitadas ao laboratório antes, durante e depois das seções de quimioterapia. ${ }^{7}$ A CPM, quando feita em uma extensão sanguínea bem confeccionada e corada, tem provado ser facilmente praticável, acurada, rápida e econômica para estimar a contagem de plaquetas. ${ }^{5}$

Apesar dos analisadores hematológicos de última geração proporcionarem maior precisão e exatidão para contar plaquetas, há situações em que eles podem gerar contagens espúrias. Independentemente da metodologia empregada pelo analisador hematológico, seja ela impedância, método ótico ou método imunológico, uma imprecisão na contagem pode ser gerada, principalmente em valores abaixo de $10 \times 10^{3} / \mu 1 .{ }^{12}$ Entretanto, as maiores causas de erros nas contagens de plaquetas podem resultar de características da amostra de sangue como, por exemplo, contagens falsamente baixas de plaquetas em consequência à coagulação parcial da amostra, à agregação plaquetária devido a uma coleta difícil, quantidade de anticoagulante inadequada, processos infecciosos, indução pelo EDTA, satelitismo plaquetário, aglutininas plaquetárias frias e plaquetas gigantes. ${ }^{4,14-19}$

A detecção de contagens de plaquetas falsamente diminuídas através da avaliação plaquetária em lâmina é muito importante, sobretudo para evitar a investigação e o tratamento desnecessários do paciente. Há exemplos em que a pseudotrombocitopenia conduziu a um diagnóstico equivocado de púrpura trombocitopênica idiopática e consequente tratamento com corticosteroides, transfusão de concentrados de plaquetas desnecessária e até mesmo esplenectomia. ${ }^{18}$
Quando se detectam contagens espúrias de plaquetas através da CPM, deve-se proceder à quantificação das mesmas por métodos alternativos que não sofram interferências. Nos casos em que a CAP está ligeiramente diminuída e se observaram agregados plaquetários na lâmina, pode-se descrever uma observação no resultado de que a contagem de plaquetas, apesar da presença de agregados plaquetários, está aparentemente normal de acordo com a avaliação microscópica em lâmina. Porém, nos casos de CAP muito baixas e com agregados plaquetários na lâmina ou microcoágulos na amostra, uma recoleta deve ser solicitada pelo laboratório e uma nova contagem deve ser realizada sem custo adicional. ${ }^{20}$

A precisão e exatidão da CAP é maior que a da CPM, porém, quando se utilizam, nas terapias transfusionais subsequentes a sangramentos pós-quimioterapia e nas transfusões profiláticas de plaquetas, limites de corte da CAP entre 5 e $20 \mathrm{x}$ $10^{3} / \mu 1$, deve-se estar ciente das limitações na precisão e acurácia das plaquetas contadas nestes patamares. Embora muitos analisadores hematológicos estejam validados para contar plaquetas em amostras com valores de $10 \times 10^{3} / \mu 1$ ou menos, a precisão da contagem nestes níveis é frequentemente baixa, com coeficientes de variação entre $22 \%$ e $66 \%$ e com contagens médias que diferem sensivelmente entre instrumentos de diferentes marcas e metodologias de contagem. ${ }^{28}$

$\mathrm{O}$ método mais recentemente desenvolvido para contagem de plaquetas utiliza anticorpos monoclonais específicos e fluorescentes contra as glicoproteínas da superfície da membrana plaquetária, CD41 (GPIIa) e CD61 (GPIIIa), conjuntamente com análise através de citometria de fluxo., $, 31,32$ Atualmente, o único analisador hematológico comercialmente disponível que oferece está tecnologia é o Cell-Dyn 4000 ou Cell-Dyn Sapphire (Abbott Diagnostics, EUA). Este método tem demonstrado ótima precisão e exatidão na contagem de plaquetas, especialmente em amostras trombocitopênicas, e tem sido proposto pela ISLH (International Society for Laboratory Hematology) como o novo método de referência. Apesar do ICSH (International Council for Standardization in Haematology) ainda reconhecer a contagem manual de plaquetas com contraste de fase como método internacional de referência, o mesmo também tem adotado o método imunológico como método de referência. . $8,30,32^{2}$

Deve-se verificar, paralelamente à CPM, a presença de plaquetas gigantes e agregados plaquetários grandes, pois eles podem ser contados como leucócitos pelos analisadores e assim gerar contagens de leucócitos falsamente elevadas e CAP falsamente diminuídas. Também é importante averiguar a presença de fragmentos eritrocitários, fragmentos citoplasmáticos de células leucêmicas, lipemia, bactérias, leveduras e micrócitos com volume próximo ao limite de corte ou threshold, que é o limiar volumétrico de distinção entre eritrócitos e plaquetas nos analisadores hematológicos, pois estes fatores podem conduzir a resultados falsamente aumentados na CAP. ${ }^{21-24} \mathrm{~A}$ avaliação microscópica das plaquetas é igualmente importante nos pacientes com trombocitose para 
procurar evidências de uma doença mieloproliferativa. ${ }^{25}$

De um modo geral, o principal erro das estimativas plaquetárias está em subestimar as contagens automatizadas pelo fato de que, em altas e baixas contagens, as plaquetas que estão sob os eritrócitos e não foram enumeradas geram maior imprecisão. Entretanto, geralmente estas diferenças não são clinicamente significativas a ponto de modificar decisões clínicas para manter ou modificar certo quimioterápico ou reavaliar a eficácia e sucesso de uma transfusão de plaquetas. ${ }^{5}$

As metodologias avaliadas neste trabalho, Figura $1 \mathrm{e}$ Tabela 1, obtiveram boa correlação estatística sugerindo que o seu uso pode ser recomendado em pacientes com os mais diversos valores de contagem de plaquetas. Embora a estimativa plaquetária pelo método alternativo tenha sido recomendada particularmente em pacientes com baixos valores de hemoglobina, este estudo, assim como o de Malok et al. (2007), ${ }^{12}$ encontrou valores discrepantes de estimativas plaquetárias em pacientes com dosagens de hemoglobina muito baixas. A análise dos dados sugeriu que valores reais de plaquetas acima de $600 \times 10^{3} / \mu \mathrm{l}$ podem ser superestimados pela metodologia de Nosanchuk, Chang \& Bennett. ${ }^{5}$

Nosanchuk et al. (1978), ${ }^{5}$ ao desenvolverem o método de estimativa plaquetária, avaliaram a influência do tamanho dos campos óticos e para isso utilizaram oculares padrão e de campo amplo. Estas, quando comparadas com aquelas, mostraram um falso aumento nas contagens de plaquetas normais e aumentadas; contudo, a diferença não foi notada em contagens menores que $30 \times 10^{3} / \mu \mathrm{l}$. Em contagens baixas, os dois sistemas óticos subestimaram a contagem plaquetária de referência na mesma proporção. Em contrapartida, conforme se aumentou a contagem de plaquetas, a superestimação aumentou acentuadamente quando oculares de campo amplo foram utilizadas. Este erro pôde ser compensado contandose oito campos visuais com oculares de campo amplo ao invés de dez como nas oculares padrão.

Neste trabalho, a correlação entre os métodos de Nosanchuk, Chang \& Bennett e Bárbara H. O'Connor foi alta $(r=0,992)$; entretanto, o valor de $p$, apesar de não indicar diferenças significativas, foi o mais próximo de 0,05 dentre as análises realizadas (Tabela 1). Isto talvez possa ser explicado pelo fato de, neste estudo, se utilizarem oculares de campo amplo para a realização de ambos os métodos. $\mathrm{O}$ método de Nosanchuk, Chang \& Bennett é padronizado para usar tais oculares, já o método de Bárbara H. O'Connor não descreveu o tipo de ocular empregado. Tem-se que quanto menor o número de campos visuais contados, maior será o valor da constante de multiplicação e vice-versa. Como no método de Bárbara H. O'Connor se contaram mais campos visuais com oculares de campo amplo, sugere-se que tenha ocorrido uma maior superestimação da contagem de plaquetas que no método de Nosanchuk, Chang \& Bennett, o que ocasionou uma maior diferença, porém não significativa, nas estimativas plaquetárias, as quais não foram corrigidas efetivamente após a multiplicação pela constante do método.
A Figura 2 mostra a relação entre a CAP e o número de plaquetas contadas em 10 campos de imersão. Este procedimento teve a finalidade de padronizar a técnica de CPM em relação aos microscópios utilizados, à região de leitura das lâminas, ao pessoal envolvido, assim como gerar um fator de correção em relação à técnica original. Sugere-se que a determinação de um fator de correção seja realizada em cada laboratório com o objetivo de melhorar a precisão e a confiabilidade das estimativas plaquetárias em lâmina. Povall et al. (2009) $)^{25}$ enumeraram plaquetas em 10 campos de 1.000x e multiplicaram a contagem por um fator igual a 109 de modo a fornecer a estimativa de plaquetas como $\mathrm{n} / 10^{9} / \mathrm{L}$. Já outro estudo utilizou como fator para estimar plaquetas em lâminas de sangue de gatos o valor de 19,1 multiplicado pelo valor médio de plaquetas por campo de 1000x de modo a obter o resultado em $\mathrm{n} \times 10^{3} / \mu 1 .^{27}$

As lâminas devem ser examinadas cuidadosamente sob aumento de 400 e 1000x para verificar a presença de agregados plaquetários, os quais geralmente se encontram no final da extensão sanguínea. ${ }^{7} \mathrm{~A}$ CPM, baseada em 8 ou 10 campos microscópicos, perde sua validade caso sejam notados agregados de plaquetas em um campo, porém é valida quando através desta observação se identifica uma causa de pseudotrombocitopenia. O reexame de uma amostra de um paciente deste estudo que mostrou valor de 4 plaquetas por campo de $1000 x$ e contagem automatizada alta revelou ter grandes agregados plaquetários no final da extensão e foi omitida das análises estatísticas.

Uma dificuldade encontrada nos laboratórios de hematologia é a de se fazerem extensões sanguíneas adequadas quando muitos profissionais estão envolvidos. Essa observação enfatiza que a realização automática de extensões sanguíneas em lâmina com a utilização de slide makers pode ajudar na confecção de lâminas adequadas em laboratórios com alto volume de amostras. Deve-se também ter cuidado ao examinar as lâminas para não confundir precipitados de corante com plaquetas, particularmente em pacientes trombocitopênicos. ${ }^{7}$

Em lâminas de pacientes com contagens de leucócitos $>100 \times 10^{3} / \mu 1$ observa-se uma dificuldade em se obter uma distribuição adequada das plaquetas, pois muitas delas podem ficar sob os leucócitos, tornando, desta forma, a estimativa não factível. ${ }^{7}$

Neste estudo não foi encontrada nenhuma estimativa plaquetária alta com contagem automatizada baixa. Isto pode ser considerado como um fator de segurança para a conduta terapêutica de pacientes em quimioterapia.

Nosanchuk et al. (1978) $)^{5}$ recomendam repetir contagens automatizadas somente quando a CPM discordar da CAP em mais de $100 \%$ em contagens até $30 \times 10^{3} / \mu 1$, em mais de $75 \%$ em contagens entre 30 e $60 \times 10^{3} / \mu 1$ e em mais de $50 \%$ em contagens superiores a $60 \times 10^{3} / \mu 1$. Este procedimento, além de servir de controle interno da qualidade, pode reduzir consideravelmente o número de repetições de CAP realizadas 
rotineiramente nos laboratórios, promovendo em muitos casos uma economia significativa.

Recomenda-se que a contagem automatizada de plaquetas e a contagem de plaquetas por microscopia sejam utilizadas conjuntamente, sobretudo por acrescentar mais qualidade e confiabilidade às contagens. Embora a estimativa plaquetária em lâmina não seja tão precisa como as contagens automatizadas, ela tem se mostrado clinicamente útil. ${ }^{5-7,12,26,27}$

\begin{abstract}
The platelet blood smear is an important tool in the evaluation of platelet disorders because it adds useful information to data provided by hematology analyzers. Furthermore, it serves as a tool for the internal quality control of automated counts. The aim of this work was to compare four manual methods of estimating platelets and to correlate the results with automated platelet counts. This study may also assist in the validation of these methods. The methodologies evaluated in this work were the modified Fonio method, the Barbara H. O'Connor method, the Nosanchuk Chang \& Bennett method and an alternative method based on the patient's hemoglobin. All methodologies had good correlation coefficients both when compared with the other methods tested and with automated platelet counts suggesting that their use can be recommended for the evaluation of the platelet status of any patient. Rev. Bras. Hematol. Hemoter.
\end{abstract}

Key words: Platelet blood smear evaluation; platelet count; quality control; thrombocytopenia; thrombocytosis.

\section{Referências Bibliográficas}

1. Peerschke EI. The laboratory evaluation of platelet dysfunction. Clin Lab Med. 2002;22(2):405-20.

2. Bizzozero G. Uber einer neuen farmbestandteil des blutes un dessen rolle bei der thrombose und der blutgerinnung. Virchows Arch Pathol Anat Physiol. 1882;90:261-332.

3. Hoffman R, Benz EJ, Shattil SJ, Furie B, Silberstein LB, McGlave $\mathrm{P}$, et al. Hematology. Basic Principles and Practice. Churchill Livingstone Elsevier: Philadelphia, 5.ed., 2009.

4. Moreno A, Menke D. Assessment of platelet numbers and morphology in the peripheral blood smear. Clin Lab Med. 2002; 22(1):193-213, vii.

5. Nosanchuk JS, Chang J, Bennett JM. The analytic basis for the use of platelet estimates from peripheral blood smears. Laboratory and clinical applications. Am J Clin Pathol. 1978;69(4):383-7.

6. Abbey AP, Belliveau RR. Enumeration of platelets. Am J Clin Pathol. 1978;69(1):55-6.

7. Bell A, Neely CL. Smear platelet counts. South Med J. 1980; 73 (7):899-901.

8. O'Connor BH. Color Atlas and Instruction Manual of Peripheral Blood Cell Morphology. Williams \& Wilkins: Pennsylvania, $1^{a}$ ed., 1984.

9. Bain BJ. Blood cells. A practical guide. Blackwell Publishing Ltd: Oxford, $4^{\mathrm{a} e d .,} 2006$.

10. van der Meer W, MacKenzie MA, Dinnissen JW, de Keijzer MH. Pseudoplatelets: a retrospective study of their incidence and interference with platelet counting. J Clin Pathol. 2003;56(10):772-4.

11. Fonio A. Ueber ein neues verfahren der Blutplättchenzahlung. Deut Ztschr Chir. 1912;117:176.
12. Malok M, Titchener EH, Bridgers C, Lee BY, Bamberg R. Comparison of two platelet count estimation methodologies for peripheral blood smears. Comparison of two platelet count estimation methodologies for peripheral blood smears. Clin Lab Sci. 2007;20(3):154-60.

13. Segal HC, Briggs C, Kunka S, Casbard A, Harrison P, Machin SJ, et al. Accuracy of platelet counting haematology analysers in severe thrombocytopenia and potential impact on platelet transfusion. Br J Haematol. 2005;128(4):520-5.

14. Dusse LMS, Vieira LM, Carvalho MG. Pseudotrombocitopenia. J Bras Patol Med Lab. 2004;40(5):321-24.

15. Greipp PR, Gralnick HR. Platelet to leukocyte adherence phenomena associated with thrombocytopenia. Blood. 1976;47(3):513-21.

16. Hsieh AT, Chao TY, Chen YC. Pseudothrombocytopenia associated with infectious mononucleosis. Arch Pathol Lab Med. 2003; 127 (1):e17-8.

17. Kjeldsberg CR, Swanson J. Platelet Satellitism. Blood. 1974;43 (6):831-6.

18. Payne BA, Pierre RV. Pseudothrombocytopenia: a laboratory artifact with potentially serious consequences. Mayo Clin Proc. 1984;59(2):123-5

19. von Ahsen N, Ehrlich B, Scott CS, Riggert J, Oellerich M. Cryoglobulins interfere with platelet counts by optical and impedance methods but not with the CD61 immunoplatelet count. Clin Chem. 2001;47(10):1858-60.

20. Oliveira RAG. Hemograma: como fazer e interpretar. Livraria Médica Paulista Ltda: São Paulo, $1^{\text {a }}$ ed., 2007.

21. Akwari AM, Ross DW, Stass SA. Spuriously elevated platelet counts due to microspherocytosis. Am J Clin Pathol. 1982;77(2):220-1.

22. Cantero M, Conejo JR, Jiménez A. Interference from lipemia in cell count by hematology analyzers. Clin Chem. 1996;42(6 Pt 1):987-8.

23. Dalal BI, Brigden ML. Artifacts that may be present on a blood film. Clin Lab Med. 2002;22(1):81-100, vi.

24. Gloster ES, Strauss RA, Jimenez JF, Neuberg RW, Berry DH, Turner EJ. Spurious elevated platelet counts associated with bacteremia. Am J Hematol. 1985;18(3):329-32.

25. Bain BJ. Diagnosis from the blood smear. N Engl J Med. 2005; 353(5):498-507.

26. Povall A, Kendrick CJ. Estimated platelet and differential leucocyte counts by microscopy, Sysmex XE-2100 and CellaVisionTM DM96. N Z J Med Lab Sci. 2009;63(1):3-10.

27. Séverine T, Cripps PJ, Mackin AJ. Estimation of platelet counts on feline blood smears. Vet Clin Pathol. 1999;28(2):42-45.

28. Lewis SM, Bain BJ, Bates I. Dacie and Lewis Practical Haematology. 10. ed., Philadelphia: Churchill Livingstone Elsevier, 2006.

29. Ault KA. The clinical utility of flow cytometry in the study of platelets. Semin Hematol. 2001;38(2):160-8.

30. International Council for Standardization in Haematology Expert Panel on Cytometry; International Society of Laboratory Hematology Task Force on Platelet Counting. Platelet counting by the RBC/platelet ratio method. A reference method. Am J Clin Pathol. 2001;115(3):460-4.

31. Harrison P, Horton A, Grant D, Briggs C, MacHin S. Immunoplatelet counting: a proposed new reference procedure. Br J Haematol. 2000;108(2):228-35.

32. Briggs C, Harrison P, Machin SJ. Continuing developments with the automated platelet count. Int J Lab Hematol. 2007;29 (2): 77-91.

Avaliação: Editor e dois revisores externos

Conflito de interesse: sem conflito de interesse

Recebido: 02/06/2009

Aceito após modificações: 13/08/2009 